\title{
Ciberbullying - Nueva tecnología electrónica al servicio del acoso escolar en alumnos de dos distritos de Lima, Perú
}

Ciberbullying - New electronic technology for school bullying in two Lima-Peru districts

\author{
Miguel Oliveros ${ }^{1, a, b}$, Isabel Amemiya ${ }^{2, a}$, Yolanda Condorimay ${ }^{3, c, d}$, Ricardo Oliveros ${ }^{4, e, f}$, \\ Armando Barrientos ${ }^{5,9}$, Bruno Emilio Rivas ${ }^{6, \mathrm{~h}}$ \\ Docente del Departamento Académico de Pediatría, Facultad de Medicina, Universidad Nacional Mayor de San Marcos, Lima, Perú. \\ ${ }^{2}$ Docente del Departamento Académico de Medicina Preventiva y Salud Pública, Facultad de Medicina, Universidad Nacional Mayor de San \\ Marcos, Lima, Perú. \\ ${ }^{3}$ Universidade Federal da Bahia, Brasil. \\ ${ }^{4}$ Docente del Departamento Académico de Psicología, Facultad de Psicología, Universidad Nacional Mayor de San Marcos, Lima, Perú. \\ ${ }^{5}$ Unidad de Investigación, Instituto de Salud del Niño, Lima, Perú. \\ ${ }^{6}$ Educador, ISTP María Rosario Araoz Pinto. \\ ${ }^{\mathrm{a}}$ Médico Pediatra; ${ }^{\mathrm{b}}$ Doctor en Medicina; ${ }^{\mathrm{C}}$ Enfermera; ${ }^{\mathrm{d}}$ Doctora en Enfermería; ${ }^{\mathrm{P}}$ Psicólogo; ${ }^{\mathrm{f}} \mathrm{Mg}$ en Psicología; ${ }^{9}$ Ingeniero estadístico; ${ }^{\mathrm{h}}$ Educador.
}

\begin{abstract}
Resumen
Planteamiento del problema: Los medios de información masiva frecuentemente comunican casos de violencia escolar expresada como bullying y con menor frecuencia ciberbullying. Objetivos: Conocer las características del ciberbullying en escolares de colegios nacionales y privados de 2 distritos de Lima. Diseño: Estudio transversal analítico, con método de encuesta. Instituciones: Facultad de Medicina, Universidad Nacional Mayor de San Marcos, Lima, Perú; y Universidade Federal da Bahia, Brasil. Participantes: Estudiantes de primaria y secundaria. Metodología: Se realizó una encuesta a 2596 estudiantes de $5^{\circ}$ de primaria a $5^{\circ}$ de secundaria, de dos colegios privados y seis colegios nacionales. El instrumento de Rosario Ortega fue modificado y validado con la prueba de Cronbach $(0,872)$. Contó con la aprobación del Comité de Ética y de los directivos institucionales. Se contó con el asentimiento de los participantes y el respeto de las consideraciones éticas de la investigación en seres humanos. Principales medidas de resultados: Caracteristicas del ciberbullying. Resultados: Se encontró ciberbullying en $27,7 \%$ de los alumnos encuestados: $21 \%$ en colegios nacionales y $41,2 \%$ en privados. Los agresores y las víctimas por celular y por internet fueron más frecuentes en los colegios privados. Tener celular, computadora en el cuarto, acceso a internet fuera de casa y trabajo remunerado fueron factores de riesgo de ciberbullying. Los agresores por celular y por internet eran preferentemente de sexo masculino y del nivel secundario. Contar con celular se relacionó con agresión por este medio, mientras que acceder a internet fuera de casa facilitó la agresión por internet. Conclusiones: La presencia de ciberbullying resultó muy alta, por lo que debe ser considerado un problema de salud pública; es más frecuente en los colegios privados que en los nacionales, y se aprecia desde el nivel primario.
\end{abstract}

Palabras clave: Intimidación, adolescente, estudiantes, violencia, agresión, acecho, ciberbullying.

Abstract

Problem: The mass media often reports cases of school violence expressed as bullying and less frequently as cyberbullying. Objectives: To determine cyberbullying characteristics in public and private school children from two Lima districts as well as factors associated with electronic aggression. Design: Cross-sectional survey. Setting: Faculty of Medicine, Universidad Nacional Mayor de San Marcos, Lima, Peru; and Universidade Federal da Bahia, Brasil. Participants: Elementary and high school students. Methods: Cross-sectional survey in 2596 5th grade of elementary school to 5th grade of high school students from two private and six public schools. The Rosario Ortega measuring instrument was modified and validated with Cronbach test (0.872). The study was approved by the Ethics Committee and school principals. It also received the assent of participants and was respectful of ethical considerations in human research. Main outcome measures: Cyberbullying characteristics. Results: Cyberbullying was reported in $27.7 \%$ of the students surveyed, $21 \%$ in public schools and $41.2 \%$ in private schools. Aggressors and victims with cell phone and internet were more common in private schools. To have cell phones, computers in their rooms, internet access out of home and paid work were risk factors for cyberbullying. Cell phone and internet attackers were predominantly male and high schoolers. Having a cell phone was associated with aggression with this device, and outdoor internet access facilitated internet aggression. Conclusion: Presence of cyberbullying was very high, and should be considered a public health problem. It was more common in private schools than in public schools, and existed beginning at primary level.

Key words: Bullying, adolescent, students, violence, aggression, stalking, cyberbullying.

An Fac med. 2012;73(1):13-8 


\section{INTRODUCCIÓN}

La violencia escolar es un problema de salud pública en el mundo entero ${ }^{(1)}$. Desde que en la década de 1970 el noruego Olweus llamara la atención sobre su prevalencia y los daños que ocasiona en víctimas y agresores, son numerosas las publicaciones que se han efectuado ${ }^{(2-6)}$. Es interesante mencionar que nuestro insigne vate César Vallejo, en 1932, se había referido anteriormente a este problema, en su cuento Paco Yunque ${ }^{(3)}$.

En el Perú, también se han realizado publicaciones sobre acoso, violencia escolar o bullying en colegios estatales y privados en zonas donde existió terrorismo, habiéndose encontrado una prevalencia alarmante, cercana al $50 \%{ }^{(4-6)}$.

Con los avances de la tecnología electrónica, ha surgido una nueva variedad de violencia escolar utilizando tecnología de comunicación e información (TICS). El uso de celulares e internet ha tenido acogida universal entre escolares y jóvenes. El uso incorrecto de estos medios informáticos manifestado por molestias, agresión o acoso es llamado ciberbullying o ciberacoso ${ }^{(7-10)}$. El primer trabajo de investigación sobre este problema se llevó a cabo el año 2000, en Estados Unidos ${ }^{(11)}$, con una encuesta telefónica a gran escala, encontrándose que $6 \%$ de adolescentes era agredido por internet, el $33 \%$ lo era a través de mensajería instantánea, $32 \%$ en salas de Chat y $19 \%$ usando correo electrónico (e-mail).

Se define ciberbullying o ciberacoso como todo acto agresivo e intencionado llevado a cabo de manera repetida y constante a lo largo del tiempo, mediante el uso de formas de contacto electrónicas por parte de un grupo o de un individuo contra una víctima que no puede defenderse fácilmente ${ }^{(8)}$.

Dentro de los variados tipos de ciberacoso se menciona ocho ${ }^{(12)}$ :

- Provocación incendiaria, discusión que se inicia generalmente en internet y que aumenta de tono y menu-

Tabla 1. Características del ciberbullying y uso de tecnologías electrónicas según colegios privados y nacionales $(n=2596)$.

\begin{tabular}{cccc} 
Variables & Colegios nacionales $(\%)$ & Colegios privados $(\%)$ & $p$ \\
Sexo masculino & $875(50,7)$ & $473(54,8)$ & $0,006^{*}$ \\
Ciberbullying & $358(21,0)$ & $352(41,2)$ & $0,000^{*}$ \\
Tiene celular & $1097(63,8)$ & $747(86,8)$ & $0,000^{*}$ \\
Agresión por celular & $112(6,5)$ & $65(7,6)$ & $\mathrm{NS}$ \\
Víctima por celular & $179(10,4)$ & $127(14,8)$ & $0,002^{*}$ \\
Tiene computadora & $1042(62,2)$ & $842(98,9)$ & $0,000^{*}$ \\
Computadora en su cuarto & $405(24,2)$ & $308(36,1)$ & $0,000^{*}$ \\
Internet fuera de casa & $1108(69,1)$ & $569(66,2)$ & $\mathrm{NS}$ \\
Agresión por internet & $146(8,5)$ & $89(10,3)$ & $\mathrm{NS}$ \\
Víctimas por internet & $330(19,2)$ & $44(23,1)$ & $0,003^{*}$ \\
\hline
\end{tabular}

${ }^{*}=p<0.05$, prueba $Z$ de diferencia de proporciones.

dean los insultos con rapidez como un incendio.

- Hostigamiento, envío repetido de mensajes desagradables.

- Denigración, enviar o 'colgar' en la red rumores sobre otra persona, para dañar su reputación o sus amistades.

- Suplantación de la personalidad, hacerse pasar por la víctima en el ciberespacio o usar su celular para, suplantando, increpar a sus amigos.

- Violación de la intimidad, compartir con terceras personas los secretos, informaciones o imágenes embarazosas de alguien en la red.

- Juego sucio, hablar con alguien sobre secretos o información incómoda, para después compartirla en contacto con otras personas.

- Exclusión, excluir a alguien de un grupo online en forma deliberada y cruel.
- Ciberacoso, palabras amenazantes o denigrantes que buscan infundir miedo o intimidar.

La información creciente y escasa publicación en nuestro país motivó nuestro interés en investigar esta problemática. La presente publicación tiene como objetivo analizar las características del ciberbullying en escolares de $5^{\circ}$ de primaria a $5^{\circ}$ de secundaria de colegios nacionales y privados de dos distritos de Lima.

\section{MÉTODOS}

Realizamos una investigación transversal analítica con el método de encuesta, técnica de cuestionario anónimo y auto aplicado en escolares de $5^{\circ}$ de primaria a $5^{\circ}$ de secundaria de colegios nacionales y privados.

Se utilizó el instrumento de Rosario Ortega ${ }^{(10)}$, el mismo que fue adaptado de acuerdo a nuestra realidad y poste-

Tabla 2. Factores de riesgo asociados a cyberbullying. Análisis bivariado.

\begin{tabular}{cccc} 
Variables & p & OR & IC $95 \%$ \\
Tenencia de celular* & 0,000 & 1,547 & $1,264-1,894$ \\
Computadora en su cuarto* & 0,000 & 1,550 & $1,252-1,918$ \\
Acceso a internet fuera de casa * & 0,003 & 1,463 & $1,140-1,879$ \\
Trabajo remunerado* & 0,001 & 1,648 & $1,249-2,175$ \\
\hline${ }^{*}{ }^{*}<0,05$. & &
\end{tabular}

${ }^{*} p<0,05$. 
Tabla 3. Variables asociadas a la presencia de ciberbullying. Análisis multivariado exploratorio $(n=2596)$.

\begin{tabular}{cccc} 
Variables & $p$ & OR & IC 95\% \\
Acceso a internet fuera de casa & 0,022 & 1,419 & $1,051-1,915$ \\
Conoces otra forma de acoso & 0,006 & 1,499 & $1,125-1,998$ \\
Trabajo remunerado & 0,000 & 1,761 & $1,301-2,383$ \\
\hline
\end{tabular}

riormente sometido a validación cualitativa por jueces; el alfa de Cronbach fue de 0.872. La encuesta contó con 30 preguntas cerradas, la mayor parte de tipo Likert. Se elaboró una guía para ser usada por los facilitadores y favorecer la homogeneidad de los resultados. El llenado de la encuesta tuvo una duración promedio de 45 minutos.

La investigación se llevó a cabo en seis colegios nacionales y dos privados, de los distritos de Pueblo Libre y Jesús María. Se incluyó 2596 alumnos comprendidos entre el quinto de primaria y quinto de secundaria. Participaron todos los alumnos presentes el día de la encuesta, la cual fue realizada simultáneamente en todas las aulas de cada colegio.

En uno de los colegios nacionales donde la población de alumnos fue grande, se tomó una muestra significativa y aleatoria de aulas; la encuesta se efectuó en tres días diferentes, de acuerdo a los años de estudio. Todos los alumnos contaron con un facilitador por aula, para resolver las dudas e interrogantes.

El Comité de Ética en Investigación, de la Facultad de Medicina de la Universidad Nacional Mayor de San Marcos, aprobó el presente trabajo. Los directores de los colegios participantes autorizaron la ejecución. Los resultados serán divulgados a las instituciones educativas y en medios de divulgación científica. Se respetó la autonomía y anonimato de los alumnos al permitirles la participación voluntaria. Se enfatizó la beneficencia al fortalecer valores como respeto, tolerancia y asertividad. Se cuidó la justicia social haciendo el estudio en colegios estatales y privados.

Se realizó análisis descriptivo e inferencial, valorando la presencia de ciberbullying en los colegios privados y nacionales y los factores de riesgo asociados a agresión por celular y por internet. Se hizo análisis univariados, de contingencia, pruebas estadísticas de asociación y exploración utilizando análisis multivariado.

Se empleó el paquete estadístico SPSS 15.0 para el procesamiento de los datos.

\section{RESULTADOS}

Presentamos los datos globales obtenidos de colegios privados y nacionales sobre la presencia de ciberbullying a través de celular y de internet, de acuerdo al sexo, tenencia de computadora en su cuarto y victimización. Un total de 1349 (52\%) alumnos fueron de sexo masculino y 1247 (48\%) de sexo femenino. Se relacionó el ciberbullying con algunas variables de la población estudiada utilizando chi cuadrado y regresión logística (tabla 1).

Las variables que destacaron fueron la mayor presencia de ciberbullying, tenencia de celular, tenencia de computadora y computadora en su cuarto, en colegios privados.

En el análisis bivariado encontramos que poseer celular, tener computadora en su cuarto, tener acceso a otra fuente de tecnología electrónica fuera de casa y desempeñar un trabajo con remuneración fueron un riesgo de ciberbullying (tabla 2).

Tabla 4. Factores de riesgo de la agresión por celular. Análisis bivariado.

\begin{tabular}{|c|c|c|c|c|c|}
\hline Variables & n/total (\%) & $\mathrm{Chi}^{2}$ & $p$ & OR & IC 95\% \\
\hline $\begin{array}{c}\text { Sexo masculino } \\
\text { Agresor por celular } \\
\text { No agresor por celular }\end{array}$ & $\begin{array}{c}69 / 107(64,5) \\
872 / 1736(50,2)\end{array}$ & $\begin{array}{l}\text { Yates } \\
=7,636\end{array}$ & 0,006 & 1,799 & $1,198-2,703$ \\
\hline $\begin{array}{c}\text { Nivel secundario } \\
\text { Agresor por celular } \\
\text { No agresor por celular }\end{array}$ & $\begin{array}{c}96 / 108(88,9) \\
1148 / 1741(65,9)\end{array}$ & $\begin{aligned} & \text { Yates } \\
= & 23,299\end{aligned}$ & 0,000 & 4,132 & $2,249-7,592$ \\
\hline $\begin{array}{c}\text { Tiene celular } \\
\text { Agresor por celular } \\
\text { No agresor por celular }\end{array}$ & $\begin{array}{c}82 / 107(76,6) \\
1119 / 1728(64,8)\end{array}$ & $\begin{array}{l}\text { Yates } \\
=5,755\end{array}$ & 0,016 & 1,785 & $1,129-2,840$ \\
\hline $\begin{array}{l}\text { Tiene computadora } \\
\text { Agresor por celular } \\
\text { No agresor por celular }\end{array}$ & $\begin{array}{c}67 / 105(63,8) \\
1086 / 1676(64,8)\end{array}$ & $\begin{array}{l}\text { Yates } \\
=5,775\end{array}$ & NS & 0,958 & $0,635-1,444$ \\
\hline $\begin{array}{l}\text { Desaprueba en estudios } \\
\text { Agresor por celular } \\
\text { No agresor por celular }\end{array}$ & $\begin{array}{c}7 / 91(7,7) \\
135 / 1580(8,5)\end{array}$ & $\begin{aligned} & \text { Yates } \\
= & 23,299\end{aligned}$ & NS & 0,008 & $0,404-1,967$ \\
\hline
\end{tabular}


Tabla 5. Factores de riesgo de la agresión por internet. Análisis bivariado.

\begin{tabular}{|c|c|c|c|c|c|}
\hline Variables & n / total (\%) & $\mathrm{Chi}^{2}$ & $\mathrm{p}$ & $\mathrm{OR}$ & IC $95 \%$ \\
\hline \multicolumn{6}{|l|}{ Sexo masculino } \\
\hline Agresor por internet & $133 / 165(80,6)$ & Yates & 0.000 & 4,421 & $2,971-6,580$ \\
\hline No agresor por internet & $815 / 1682(48,5)$ & $=60,895$ & & & \\
\hline \multicolumn{6}{|l|}{ Nivel secundario } \\
\hline Agresor por internet & $144 / 166(86,7)$ & Yates & 0,000 & 3,502 & $2,211-5,546$ \\
\hline No agresor por internet & $1099 / 1687(65,1)$ & $=30,965$ & & & \\
\hline \multicolumn{6}{|l|}{ Tiene celular } \\
\hline Agresor por internet & $113 / 166(68,1)$ & Yates & NS & 1,158 & $0,823-1,630$ \\
\hline No agresor por internet & $1084 / 1673(64,8)$ & $=0,577$ & & & \\
\hline \multicolumn{6}{|l|}{ Tiene computadora } \\
\hline Agresor por internet & $111 / 161(68,9)$ & Yates & NS & 1,216 & $0,858-1,723$ \\
\hline No agresor por internet & $1050 / 1625(64,6)$ & $=1,024$ & & & \\
\hline \multicolumn{6}{|l|}{ Tiene computadora en cuarto } \\
\hline Agresor por internet & $43 / 161(26,7)$ & Yates & NS & 1,049 & $0,758-1,579$ \\
\hline No agresor por internet & $406 / 1625(25,0)$ & $=0,149$ & & & \\
\hline \multicolumn{6}{|l|}{ Usa internet fuera de casa } \\
\hline Agresor por internet & $119 / 152(78,3)$ & Yates & 0,012 & 1,689 & $1,132-2,519$ \\
\hline No agresor por internet & $1072 / 1574(68,1)$ & $=6,252$ & & & \\
\hline \multicolumn{6}{|l|}{ Desaprueba en estudios } \\
\hline Agresor por internet & $19 / 140(13,6)$ & Yates & 0,041 & 1,774 & $1,058-2,975$ \\
\hline No agresor por internet & $121 / 1537(8,1)$ & $=4,167$ & & & \\
\hline
\end{tabular}

En el análisis multivariado exploratorio (tabla 3) se confirmó que tener un acceso de internet fuera de casa y un trabajo remunerado fueron factores de riesgo de ciberbullying. Además, apareció como nueva variable relacionada el conocer otra forma de acoso.

La frecuencia global de ciberbullying fue $27,7 \%$ (710 casos). Las víctimas de agresión por celular fueron $10,8 \%$ en varones y $13,1 \%$ en mujeres (chi ${ }^{2} 3,23$ $p=0,072)$. Los varones aceptaron ser agresores por este medio con mayor asiduidad $(7,3 \%)$ que las mujeres, $4,25 \%$ (chi ${ }^{2}$ 7,636; p=0,006; OR=1,799; $\mathrm{IC}=1,198-2,703)$.

Con respecto a internet, ser agresor también primó entre los varones (14\%) sobre las mujeres, 3,6\% (chi ${ }^{2}$ 60,895; $\mathrm{p}=0,000 ; \quad \mathrm{OR}=4,421 ; \quad \mathrm{IC}=2,971$. $6,580)$. Ser varón resultó ser un factor de protección para las víctimas, pues los varones se señalaron como víctimas menos frecuentemente $(18 \%)$ que las mujeres, 22,7\%, $\left(\right.$ chi $^{2} 6,181 ; \mathrm{p}=0,013$; $\mathrm{OR}=0,774 ; \mathrm{IC}=0,593-0,935)$.

El sexo masculino, estudiar en el nivel secundario y la tenencia de celular fueron factores de riesgo de agresión por celular (tabla 4).

El sexo masculino, estudiar en el nivel secundario, uso de internet fuera de casa y desaprobar en los estudios fueron factores de riesgo de agresión por internet (tabla 5).

El sexo masculino también se encontró que fue un factor de riesgo de agresión por internet en el nivel de educación primaria y secundaria, tanto en el colegio privado como en el nacional.

\section{DISCUSIÓN}

Los cambios que nos ha traído la modernidad han trastocado los estilos de vida tradicionales. Los colegios, considerados instituciones de socialización y convivencia pacífica, han perdido la seguridad que brindaban, y en ellos muchas veces se encuentran los alumnos desprotegidos e indefensos. El uso de celulares desde temprana edad y la familiaridad con que utilizan internet, se han convertido en armas o instrumentos de agresión, actitud que se emplea desconociendo o minimizando los riesgos que conlleva el acoso escolar.

Entre las tecnologías de comunicación e información (TICs) se encuentran el correo electrónico, mensajes con celulares, mensajería instantánea y videos, los que son empleados para amenazar, vejar, chantajear, discriminar o difamar en forma repetida y hostil, siempre con el propósito de ocasionar daño a las víctimas ${ }^{(7,12-15)}$.

El hallazgo de 27,7\% de ciberbullying o ciberacoso es preocupante, más aún, encontrar este problema, con más frecuencia, en colegios privados que en nacionales. También, los agresores y víctimas son más frecuentes en colegios privados. Esta agresión ha sido mucho más frecuente a través de internet que con celular.

El internet es usado con más frecuencia desde su casa, colegio o cabina de chateo. Ese último lugar cuesta muy poco dinero, permite que se puedan reunir amigos para disfrutar en grupo, constituyéndose en el lugar preferido por la libertad y falta de supervisión existente. A los estudiantes les agrada 
también utilizar Play Station y Nintendo, juegos que estimulan la violencia ${ }^{(10-18)}$. En nuestro estudio, los alumnos de colegios nacionales y privados usaron con frecuencia el internet fuera de casa, exponiéndolos a riesgo de uso inadecuado de este medio. El uso incorrecto de internet se ha constituido en importante factor de riesgo en los análisis de asociación y multivariados.

Hemos encontrado en nuestro estudio que los alumnos con mejor posición social disponen con mayor frecuencia de celulares y de internet en sus cuartos o dormitorios personales, desde los cuales, sin control parental, pueden emplearlas a su libre albedrío. Es opinión general del grupo investigador que es necesaria la supervisión de los menores, y para ello las computadoras con internet deben estar en ambientes compartidos por la familia ${ }^{(18)}$. Algunos alumnos que tienen que realizar en sus horas fuera del colegio algún trabajo remunerado están más expuestos al ciberbullying, probablemente por el estigma generado por su condición de mayor pobreza, fatiga y pobre rendimiento escolar, a consecuencia de la carga laboral, aunado al hecho de la baja remuneración y desprotección de parte de sus familiares.

Finkelhorn, en Estados Unidos (11), con una encuesta telefónica a gran escala encontró que el 6\% de adolescentes era agredido por internet, 33\% a través de mensajería instantánea, $32 \%$ en salas de chat y 19\% usando correo electrónico. Li ${ }^{(9)}$ estudió 177 adolescentes en Canadá, 80 chicos y 97 chicas, y una cuarta parte de ellos manifestó haber estado implicados en ciberbullying. Smith y col ${ }^{(8)}$ han detectado que 15,6\% del alumnado declaró haber sido víctima de ciberbullying una a dos veces, mientras que 6,6\% dijo haberlo sufrido una o más de una vez a la semana.

En España ${ }^{(14)}$, en el último estudio del Defensor del Pueblo, se detectó 5,5\% de ciber víctimas; de ellas, 5,1\% lo era en forma esporádica (menos de una vez a la semana) y $0,4 \%$ con una frecuencia superior. Se encontró porcentajes similares entre los agresores: $5,4 \%$ en la muestra total, de los cuales $4,8 \%$ lo era en forma eventual y $0,6 \%$ de forma frecuente.

En los últimos años, hay tendencia al aumento del ciberacoso. Así, Brugees ${ }^{(15)}$, en EE UU, encontró que 38,3\% de la muestra (3 141 chicas) había sido acosada on line. Raskauskas ${ }^{(13)}$, en el mismo país, señala que 48,8\% de los encuestados estaría implicado en ciberbullying como víctima y 21,4\% como agresor. También, se ha encontrado que las víctimas de bullying tradicional tienen riesgo de ser víctimas de ciberacoso; situación similar ocurre en el caso de los agresores. Jo Roskam (22), al investigar el ciberbullying en 1000 estudiantes de Illinois, encontró que 332 informaron haber sido agredidos, la mayor parte con apodos, expansión de rumores, humillación y amenazas. Los tipos de agresión empleados fueron mensajes de texto en $24,5 \%$, mensajes utilizando la voz en 15,4\% y fotos en 9,9\%. Smith P ${ }^{(23)}$, en Londres, señala que el ciberbullying más frecuente ocurre fuera del colegio, mientras que en el colegio $14,3 \%$ fue agredido con celular y 5,5\% por correo electrónico. Estas cifras, en su siguiente investigación aumentaron a 25,9 y 10,9, respectivamente.

Encontramos, en contraposición a los resultados de Smith P y col ${ }^{(23)}$, cifras más altas de ciber víctimas; por internet alcanzaron $19,2 \%$ y $23,1 \%$ en colegios nacionales y privados, respectivamente, y por celular $10,4 \%$ y $14,8 \%$.

Ambos sexos participan del ciberbullying. Sin embargo, el sexo masculino es un factor de riesgo de agresión por internet y por celular, tanto en primaria como en secundaria y en colegios privados y nacionales. Pero, ser víctima por celular no se asoció al sexo y al contrario el sexo masculino resultó ser un factor de protección para ser víctima por internet. Lo que sí nos mostró fue una tendencia a que las mujeres fueran más victimas tanto por celular como por internet.
Es difícil que agresores y víctimas acepten su participación. Sin embargo, las víctimas se sinceraron con más frecuencia. Además, los agresores y víctimas por internet comunicaron más este problema que los que utilizaban el celular.

Se puede apreciar, después de la revisión de la literatura, la alta frecuencia de ciberbullying o ciberacoso, la tendencia creciente, su predominio en varones, las diversas modalidades empleadas utilizando medios electrónicos y la preferencia de agresión por internet, para preservar el anonimato ${ }^{(7-10,13-15)}$.

El ciberbullying comparte con el bullying los elementos de desequilibrio de poder, reiteración e intencionalidad, y a veces puede ser consecuencia o continuación del bullying escolar. Los daños causados son mayores que el bullying, pues internet garantiza el anonimato del agresor, convirtiéndolo en un fantasma que perturba y destroza con crueldad la vida de sus pares ${ }^{(18)}$.

El internet despierta en algunos niños y adolescentes el sentimiento de que no existen normas jurídicas o principios éticos que regulen las interacciones que se producen en la red; por ello, experimentan total libertad para lo bueno y para lo malo. La red ofrece las mismas paradojas que nos brinda la sociedad. Podemos encontrarnos con comunidades virtuales solidarias y con los más perversos agresores infantiles. Este riesgo exige a padres y maestros prevención a partir de una formación adecuada y el uso responsable de las tecnologías de información y comunicación $^{(16-20,24)}$.

Estos resultados representan solo dos distritos de Lima Metropolitana; por ello, es conveniente realizar estudios en otras instituciones educativas, nacionales y privadas.

La Asociación Alternativa Joven de Extremadura ${ }^{(16)}$ recomienda, como prevención:

- Los padres/madres deben hablar con sus hijos sobre los valores y el com- 
portamiento en sus relaciones con sus compañeros escolares.

- Deben informar a sus hijos de las consecuencias perjudiciales, tanto para la víctima como para el agresor, de llevar a cabo prácticas de 'ciberacoso'.

- Escuchar activamente a los hijos, conociendo sus problemas y preocupaciones, buscando siempre aquello que los enriquezca humanamente.

- Informarles de los aspectos negativos de las relaciones virtuales con desconocidos a través de internet.

- Prevenirles de los riesgos de divulgar online o presencialmente información personal, propia o ajena (de otros compañeros).

- Los padres/madres de menores que por sus circunstancias personales se encuentran en situación de riesgo de ser víctimas, deben estar especialmente atentos e informados sobre el maltrato escolar.

- Preguntarle si conoce algún caso de 'acoso escolar' cercano y exponerle algunas noticias o casos reales para debatir en familia.

A lo mencionado se suma la publicación reciente del Center for Safe and Responsible Internet Use (2006), que ha elaborado una guía dirigida a padres sobre ciberbullying, con la intención de poder ayudarles a prevenir que sus hijos se conviertan en víctimas de sus compañeros. Esta guía comienza narrando historias reales de niños que han sido víctimas de diversos tipos de ciberbullying, expone algunos de los riesgos de internet (contenido altamente sexual, suicidio y grupos de bandas, sectas y fomento de la violencia), analiza algunos de los motivos por los cuales los padres permanecen ajenos ante esta realidad y ofrece orientaciones de tipo preventivas y correctivas ${ }^{(21)}$.

En conclusión, encontramos que la presencia de ciberbullying en los distritos estudiados es muy alta, por lo que debe ser considerado un problema de salud pública; es más frecuente en los colegios privados que en los nacionales; se aprecia desde el nivel primario.

Se debe difundir a nivel escolar y familiar la importancia y repercusiones del ciberacoso, acompañados del diseño de estrategias de intervención iniciadas desde el nivel primario.

Se sugiere educar sobre el uso adecuado de las TICs en hogar y escuelas, a fin de prevenir y desencadenar otras formas de violencia. Es necesario incrementar el horizonte de esta investigación en otras regiones, con miras a identificar la magnitud del problema y profundizar el conocimiento empleando investigación cualitativa.

\section{AGRADECIMIENTOS}

A los directores, docentes y estudiantes de los colegios que participaron en la investigación, por su valiosa colaboración y apoyo.

\section{REFERENCIAS BIBLIOGRÁFICAS}

1. Bauer N, Herrenkohl T, Lozano P, Rivara F, Hill K, Hawkiins J. Childhood bullying involvement and exposure to intimate partner violence. Pediatrics. 2006;118(2):E235-E242.

2. Olweus D. Stability of aggressive reaction patterns in males. A review. Psychol Bull. 1979;86:852-75.

3. Vallejo C. Paco Yunque. Editorial San Marcos 2008, Lima Perú.

4. Oliveros M, Figueroa L, Mayorga G, Cano B, Quispe Y. Violencia escolar (bullying) en colegios estatales de primaria en el Perú. Rev Per Pediatría. 2008;61(4):215-20.

5. Oliveros M, Figueroa L, Mayorga G, Cano B, Quispe $Y$. Intimidación (bullying), experiencia en colegios estatales de secundaria del Perú. Rev Per Pediatría. 2009;62(1):19-29.

6. Amemiya I, Oliveros M, Barrientos A. Factores de riesgo de violencia escolar (bullying) severa en colegios privados de tres zonas de la sierra del Perú. An Fac med. 2009;70(4):255-8.

7. Campbell MA. Cyberbullying: An old problem in a new guise? Australian J Guidance Counselling. 2005;15(1):68-76.

8. Smith KP. Ciberacoso: naturaleza y extensión de un nuevo tipo de acoso dentro y fuera de la escuela. Congreso Educación Palma de Mallorca, 2006.

9. Li Q. Cyberbullying in schools: A research of gender differences. School Psychol Internat. 2006;27:15770.

10. Ortega R, Calmaestra J, Mora Merchan J. Cyberbullying. Intern J Psychol Psychologic Ther. 2008;8(2):183-92.
11. Finkelhor D, Mitchell KJ, Wolak J. Online victimization a report on a nation's youth. Alexandria VA: National Center for Missing and Exploted Children, 2000.

12. Willard N. Educators guide to Cyberbullying and Cyberthreads (14/5/2011) http//:new. csrin.org/ cyberbully/docs/cbcteducator.pdf

13. Raskaukas J, Stoltz AD. Involvement in traditional and electronic bullying among adolescents. Dev Psychol. 2007;43(3):564-75.

14. Defensor del Pueblo-UNICEF. Violencia escolar. E maltrato entre iguales en la educación secundaria obligatoria 1999-2006. Madrid: Publicaciones Oficinas del Defensor del Pueblo, 2006.

15. Brugess-Proctor A, Patchin JW. Hinduja S. Cyberbullying. The victimization of adolescent girls. En: Garcia V, Clifford J (Eds). Female crime victims: Reality reconsidered. Upper Saddle River, NJ: Prentice Hall. 2009.

16. Rivero Camberro S. Alternativa Joven de Extremadura. E-bullying o ciberacoso Nuevo modo de acoso entre iguales (14/5/2011) www.alternativa-joven. org.

17. López Herrero M, López Moreno V, Galán Martín E. Redes Sociales de Internet y adolescentes. (14/5/2011) www.websatafi.com.pdf.

18. Collell J, Escudé C. Cyberbullying. L'assetjament a través de la xarxa. Àmbits de Psicopedagogia. 2008;24:2023.

19. Pérez Murillo. Las nuevas tecnologias al servicio del acoso escolar. Innovación y Experiencias Educativas 2008, Granada. (14/5/2011) csifrevistad@ gmail.com.

20. Gallardo L. Dominio y sumisión en las aulas. (14/5/2011) www.aprendemas.com/ Reportajes/.../ Acoso\%20Escolar.pdf - España.

21. CRIU Center for Safe and Responsible Internet Use (14/5/2011) www.cyberbully.org/ consulting.php.

22. Roskamp TJ. Cyberbullying in Illinois in public schools. Thesis dissertation for Doctor of Education degree. School of Graduate Studies Western Illinois University, 2009, April.

23. Smith KP. Mahdavi J, Carvalho M, Fisher S, Russell S, Tippett N. Cyberbullying. its nature and impact in secondary school pupils. J Child Psychol Psychiatry. 2008;49(4):376-85.

24. Shariff S. Confronting cyber-bullying: what schools need to know to control misconduct and avoid legal consequences. New York: Cambridge University Press, 2009.

Trabajo recibido el 28 de octubre de 2011 y aceptado para publicación el 11 de enero de 2012.

Fuente de financiamiento:

Esta investigación fue financiada por el Vicerrectorado de Investigación de la Universidad Nacional Mayor de San Marcos, como parte del Programa de Proyectos Multidisciplinarios de Investigación.

Conflictos de interés:

Los autores declaran no tener conflictos de interés en la elaboración ni publicación de este artículo.

\section{Correspondencia:}

Dr. Miguel Oliveros Donohue

drmigueloliveros@hotmail.com 